\title{
Exploring the Molecular Mechanism of Astragali Radix-Curcumae Rhizoma against Gastric Intraepithelial Neoplasia by Network Pharmacology and Molecular Docking
}

\author{
Yuejin Ji $\mathbb{D}$, Yajun Liu, Jingyi Hu, Cheng Cheng, Jing Xing, Lei Zhu $\mathbb{D}$, and Hong Shen \\ Affiliated Hospital of Nanjing University of Chinese Medicine, Nanjing 210029, China \\ Correspondence should be addressed to Lei Zhu; zhulei5100@njucm.edu.cn and Hong Shen; shenhong999@njucm.edu.cn
}

Received 18 August 2021; Accepted 22 September 2021; Published 4 October 2021

Academic Editor: Samuel Martins Silvestre

Copyright ( 2021 Yuejin Ji et al. This is an open access article distributed under the Creative Commons Attribution License, which permits unrestricted use, distribution, and reproduction in any medium, provided the original work is properly cited.

\begin{abstract}
Background. Astragali Radix-Curcumae Rhizoma (ARCR), a classic drug pair, has been widely used for the treatment of gastric intraepithelial neoplasia (GIN) in China. However, the underlying mechanisms of this drug pair are still unknown. Thus, elucidating the molecular mechanism of ARCR for treating GIN is imperative. Methods. The active components and targets of ARCR were determined from the TCMSP database, and the differentially expressed genes related to GIN were identified from the GSE130823 dataset. The protein-protein interaction (PPI) network and ARCR-active component-target-pathway network were constructed by STRING 11.0 and Cytoscape 3.7.2, respectively. In addition, a receiver operating characteristic curve (ROC) was conducted to verify the key targets, and enrichment analyses were performed using R software. Molecular docking was carried out to test the binding capacity between core active components and key targets. Results. 31 active components were obtained from ARCR, among which 22 were hit by the 51 targets associated with GIN. Gene Ontology (GO) functional enrichment analysis showed that biological process (BP), molecular function (MF), and cellular component (CC) were most significantly enriched in response to a drug, catecholamine binding, and apical part of the cell, respectively. Kyoto Encyclopedia of Genes and Genomes (KEGG) pathway analysis indicated ARCR against GIN through regulation of neuroactive ligand-receptor interaction, nitrogen metabolism, calcium signaling pathway, chemical carcinogenesis-receptor activation, drug metabolism, gap junction, and cancers. In the PPI network, 15 potential targets were identified, of which nine key targets were proven to have higher diagnostic values in ROC. Molecular docking revealed a good binding affinity of active components (quercetin, bisdemethoxycurcumin, and kaempferol) with the corresponding targets (CYP3A4, CYP1A1, HMOX1, DRD2, DPP4, ADRA2A, ADRA2C, NR1I2, and LGALS4). Conclusion. This study revealed the active components and molecular mechanism by which ARCR treatment is effective against GIN through regulating multipathway, such as neuroactive ligand-receptor interaction, nitrogen metabolism, and calcium signaling pathway.
\end{abstract}

\section{Introduction}

Gastric cancer (GC) is one of the most common cancers worldwide, ranking fifth for incidence and fourth for mortality globally [1]. Eastern Asia, including China, has the highest incidence rate [2]. In China, the number of new cases of GC was 679,000, and the number of deaths was 498,000 in 2015 [3]. Therefore, preventing the occurrence of GC in China is an arduous and great task.

Based on Lauren classification [4], GC is divided into two major histological types, diffuse and intestinal gastric cancer
(IGC) types. Correa et al. proposed a prevailing model for the stages of IGC, wherein normal gastric mucosa progresses to chronic superficial gastritis and then to chronic atrophic gastritis (CAG), intestinal metaplasia, dysplasia, and IGC [5]. As the most serious precancerous lesion of GC, the treatment of gastric intraepithelial neoplasia (GIN) is indispensable for preventing GC. At present, the main therapies for high-grade intraepithelial neoplasia include endoscopic mucosal resection and/or endoscopic submucosal dissection. However, low-grade intraepithelial neoplasia and high-grade intraepithelial neoplasia patients 
unwilling to undergo endoscopic surgery still lack effective therapies.

Astragali Radix (ARX) and Curcumae Rhizoma (CR) are two kinds of Chinese herbal medicines, which are often chosen to treat tumor-related diseases in the form of a drug pair $[6,7]$. Astragali Radix is called Huangqi in Chinese, a representative medicine for tonifying Qi and strengthening the spleen. Curcumae Rhizoma is called Ezhu in Chinese, good at activating blood and resolving stasis. The combination of ARX and CR is mainly responsible for tonifying Qi and activating blood (Yiqi Huoxue), one of the most commonly used treatment methods for GIN in traditional Chinese medicine. Qian et al. found that Yiqi Huoxue Decoction may play a preventive role in gastric precancerous lesions by downregulating the expression of Sonic Hedgehog signaling pathway [8]. In a clinical study, Yiqi Huoxue Fang combined with quadruple therapy can improve the main clinical symptoms, enhance the secretion of gastric mucosa, improve the content of pepsinogen in serum, and reduce the level of inflammatory factors in Helicobacter pylori positive CAG patients [9]. Our previous study confirmed that Yiqi Huoxue Fang based on Astragali Radix-Curcumae Rhizoma (ARCR) has a good clinical effect on gastric precancerous lesions [10].

In order to elucidate the mechanism of ARCR in the treatment of GIN, we explored the key targets and pathways of ARCR against GIN through network pharmacology and molecular docking. The flowchart of our analysis is shown in Figure 1.

\section{Materials and Methods}

2.1. Screening the Active Components and Targets for ARCR. The active components were extracted from the Traditional Chinese Medicine Database and Analysis Platform (TCMSP) (https://tcmsp-e.com/) [11]. Oral bioavailability $(\mathrm{OB}) \geq 30 \%$ and drug-likeness $(\mathrm{DL}) \geq 0.18$ were set as the thresholds to screen the active components of ARCR in the TCMSP database and then obtain the corresponding targets for each active component. Meanwhile, other active components not retrieved were supplemented by related literature reviews. If the targets of active components cannot be obtained from the TCMSP database, the prediction of the relevant targets was conducted in SwissTargetPrediction [12]. The 2D structures of the active components were downloaded from PubChem (https://pubchem.ncbi.nlm. nih.gov/) [13]. All targets of ARCR were standardized in the UniPort database [14].

\subsection{Collection of GIN-Related Differentially Expressed Genes} (DEGs). A microarray expression profiling dataset GSE130823 was selected for exploring the DEGs in Gene Expression Omnibus (GEO) [15]. GSE130823, based on the GPL17077 platform, included 98 gastric tissue biopsy samples (47 chronic gastritis, 31 GIN, and 16 IGC). The function of normizeBetweenArray in Limma package [16] was used to normalize GSE130823, and the DEGs were identified using the Limma package in R software. Targets with the cutoff criteria of $\mid \log 2$ FoldChange $\mid>1$ and adjusted $P$ value $<0.05$ were regarded as DEGs [17].

2.3. Construction of Protein-Protein Interaction (PPI) Network and Identification of the Key Targets. The intersecting targets between ARCR and GIN were obtained by ggVennDiagram package in R software, and Veen map was drawn. Then, we imported the intersecting targets into the STRING 11.0 [18], with the species limited to "Homo sapiens" and the minimum required interaction score set as 0.900 and the disconnected nodes hid in the network. Subsequently, the PPI network was visualized and analyzed in Cytoscape 3.7.2 [19], to obtain the core functional modules by Cytoscape MCODE [20] and identify the potential key targets by CytoHubba App [21]. Receiver operating characteristic curve (ROC) analysis was performed, using IBM SPSS version 26 , to verify the diagnostic values of key targets in GIN. The expression levels of the key targets were obtained from the GSE130823 dataset. The area under ROC curve (AUC) was calculated, and the statistical standard of AUC $\geq 0.7$ and $P$ value $<0.05$ were used.

2.4. Enrichment Analysis of the Intersecting Targets. We use clusterProfiler package [22] to conduct Kyoto Encyclopedia of Genes and Genomes (KEGG) pathway analysis and Gene Ontology (GO) functional enrichment analysis in R software. A bar chart or a bubble diagram was constructed after screening out the first 15 functional categories of biological process (BP), cellular component (CC), molecular function (MF), and KEGG pathway enrichment analysis, respectively. The threshold of adjusted $P$ value $<0.05$ was considered as the screening criteria.

2.5. Network Construction of ARCR-Active ComponentIntersecting Target-KEGG Pathway. ARCR, active components, intersecting targets, and signaling pathways were introduced into Cytoscape 3.7.2 software to construct and analyze the network, in order to screen the core active components of ARCR in treating GIN.

2.6. Molecular Docking Analysis. Molecular docking analysis of the core active components of ARCR and the key targets was conducted. The molecular structures of the core active components were searched in ZINC database [23], and the $3 \mathrm{D}$ structures of the key targets were obtained in PDB database [24]. Use PYMOL to remove water molecules and ligands of the targets. Upload the PDB format files of PYMOL-processed targets and the mol2 format files of the core active components to the SwissDock platform [25] for molecular docking. Use UCSF Chimera and PYMOL to analyze and visualize the results of molecular docking.

\section{Results}

3.1. Active Components and Its Corresponding Targets of $A R C R$. Initially, obtain 89 active components of ARX and 82 active components of CR from the TCMSP database. 


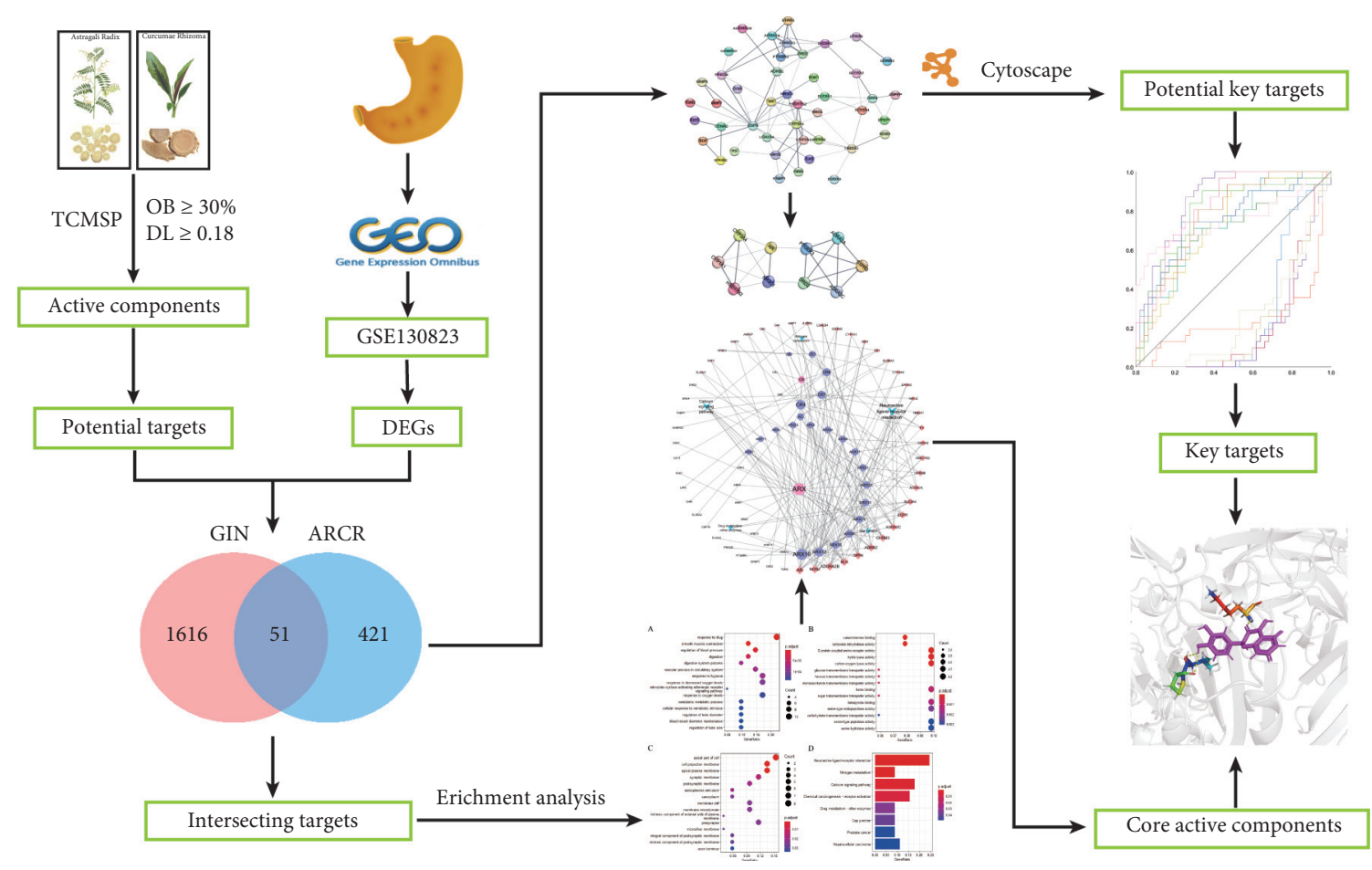

FIgURE 1: Flowchart of our study.

With the criteria of $\mathrm{OB} \geq 30 \%$ and $\mathrm{DL} \geq 0.18$, combining the related literature reviews [26-29], a total of 31 active components were identified, of which 24 were from ARX and eight were from CR (one active component was shared by ARX and CR). A total of 1101 targets were associated with the 31 active components identified in ARCR, of which 822 were associated with ARX and 279 with CR. After removing the overlapping targets, 472 targets were remaining, including 367 from ARX and 212 from CR. The active components of ARCR are shown in Table 1.

3.2. DEGs for GIN. The gastric biopsy tissues of $31 \mathrm{GIN}$ patients in the GSE130823 were used as the research object, and 47 chronic gastritis tissues were used as the control group. According to the cutoff criteria of $\mid \log 2$ FoldChange $\mid>1$ and adjusted $P$ value $<0.05$, a total of 1667 DEGs in the GSE130823 dataset were found to be dysregulated in GIN tissues by the Limma package, including 737 upregulated genes and 930 downregulated genes (Supplementary Table 1).

\subsection{PPI Network Construction and Key Targets Identification.} Using the R software to draw the Venn map between the targets of ARCR and the DEGs of GIN (Figure 2(a)), we obtained 51 intersecting targets. The STRING 11.0 was used to construct the PPI network of the intersecting targets of ARCR as related to the treatment of GIN. After hiding the isolated nodes, the PPI network contained 44 nodes and 87 edges (Figure 2(b)). One core function module was identified by Cytoscape MCODE (Figure 2(c)), and target proteins with the top 15 degree values were considered as the potential key targets in the PPI network (Table 2). The results of ROC analysis indicated that nine key targets (CYP3A4, CYP1A1, HMOX1, DRD2, DPP4, ADRA2A, ADRA2C, NR1I2, and LGALS4) had higher diagnostic values (Figure 2(d), Table 2).

3.4. GO Functional Enrichment Analysis and KEGG Pathway Analysis of Intersecting Targets. To further elucidate the biological functions of the 51 intersecting targets, GO enrichment analysis was performed in $\mathrm{R}$ software. GO functional enrichment analysis of BP obtained a total of 363 enrichment items, the targets were markedly enriched for response to drug, smooth muscle contraction, regulation of blood pressure, digestion, and digestive system process (Figure 3(a)). MF analysis obtained a total of 64 enrichment items, the targets were significantly involved in catecholamine binding, carbonate dehydratase activity, G proteincoupled amine receptor activity, hydro-lyase activity, and carbon-oxygen lyase activity (Figure 3(b)). CC analysis obtained a total of 25 enrichment items; the targets were mainly enriched in the apical part of the cell, cell projection membrane, apical plasma membrane, synaptic membrane, and postsynaptic membrane (Figure 3(c)). KEGG pathway analysis returned 8 items, including neuroactive ligand-receptor interaction, nitrogen metabolism, calcium signaling pathway, chemical carcinogenesis-receptor activation, drug metabolism, gap junction, and cancers (Figure 3(d)).

3.5. ARCR-Active Component-Intersecting Target-KEGG Pathway Network. Cytoscape 3.7.2 was used to construct the network of ARCR-active component-intersecting 
TABle 1: Active components of ARCR.

\begin{tabular}{|c|c|c|c|c|}
\hline Number & Mol ID & Molecule name & OB\% & $\mathrm{DL}$ \\
\hline CR1 & MOL000915 & $(1 \mathrm{~S}, 10 \mathrm{~S}),(4 \mathrm{~S}, 5 \mathrm{~S})$-germacrone-1(10), 4-diepoxide & 30.48 & 0.18 \\
\hline CR2 & MOL000902 & Curcumol & 103.55 & 0.13 \\
\hline CR3 & MOL000906 & Wenjine & 47.93 & 0.47 \\
\hline CR4 & MOL000940 & Bisdemethoxycurcumin & 77.38 & 0.26 \\
\hline CR5 & - & Germacrone & - & - \\
\hline CR6 & MOL000090 & Curcumin & 5.15 & 0.41 \\
\hline CR7 & - & Curdione & - & - \\
\hline ARX1 & MOL000211 & Mairin & 55.38 & 0.78 \\
\hline ARX2 & MOL000239 & Jaranol & 50.83 & 0.29 \\
\hline ARX3 & MOL000033 & Cholest-5-en-3alpha-ol & 36.23 & 0.78 \\
\hline $\mathrm{ARX} 4$ & MOL000354 & Isorhamnetin & 49.60 & 0.31 \\
\hline ARX5 & MOL000371 & 3,9-di-O-Methylnissolin & 53.74 & 0.48 \\
\hline ARX6 & MOL000378 & 7-O-Methylisomucronulatol & 74.69 & 0.30 \\
\hline ARX7 & MOL000379 & 9,10-Dimethoxypterocarpan-3-O- $\beta$-D-glucoside & 36.74 & 0.92 \\
\hline ARX8 & MOL000380 & Methylnissolin & 64.26 & 0.42 \\
\hline ARX9 & MOL000387 & Bifendate & 31.10 & 0.67 \\
\hline ARX10 & MOL000392 & Formononetin & 69.67 & 0.21 \\
\hline ARX11 & MOL000417 & Calycosin & 47.75 & 0.24 \\
\hline ARX12 & MOL000422 & Kaempferol & 41.88 & 0.24 \\
\hline ARX13 & MOL000433 & FA & 68.96 & 0.71 \\
\hline ARX14 & MOL000439 & Isomucronulatol-7,2' -di-O-glucoside & 49.28 & 0.62 \\
\hline ARX15 & MOL000442 & 1,7-Dihydroxy-3,9-dimethoxy pterocarpene & 39.05 & 0.48 \\
\hline ARX16 & MOL000098 & Quercetin & 46.43 & 0.28 \\
\hline ARX17 & MOL000374 & $5^{\prime}$-Hydroxyiso-muronulatol-2' $5^{\prime}$-di-O-glucoside & 41.72 & 0.69 \\
\hline ARX18 & MOL000398 & Isoflavanone & 109.99 & 0.30 \\
\hline ARX19 & MOL000438 & (3R)-3-(2-Hydroxy-3,4-dimethoxyphenyl) chroman-7-ol & 67.67 & 0.26 \\
\hline ARX20 & MOL000407 & Astragaloside IV & 22.5 & 0.15 \\
\hline ARX21 & MOL000401 & Astragaloside I & 46.79 & 0.11 \\
\hline ARX22 & MOL000403 & Astragaloside II & 46.06 & 0.13 \\
\hline ARX23 & - & 2-(Chloromethyl)-4-(4-nitrophenyl)-1,3-thiazole & - & - \\
\hline $\mathrm{AC}$ & MOL000296 & Hederagenin & 36.91 & 0.75 \\
\hline
\end{tabular}

CR1-CR7 represent the active components of CR; ARX1-ARX23 represent the active components of ARX; and AC is the active components shared by ARX and CR. OB: oral bioavailability; DL: drug-likeness.

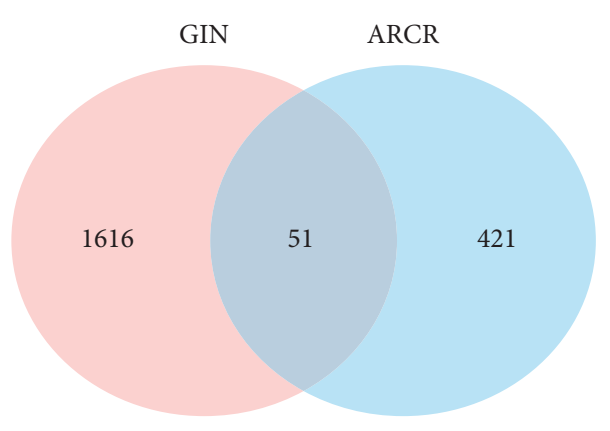

(a)

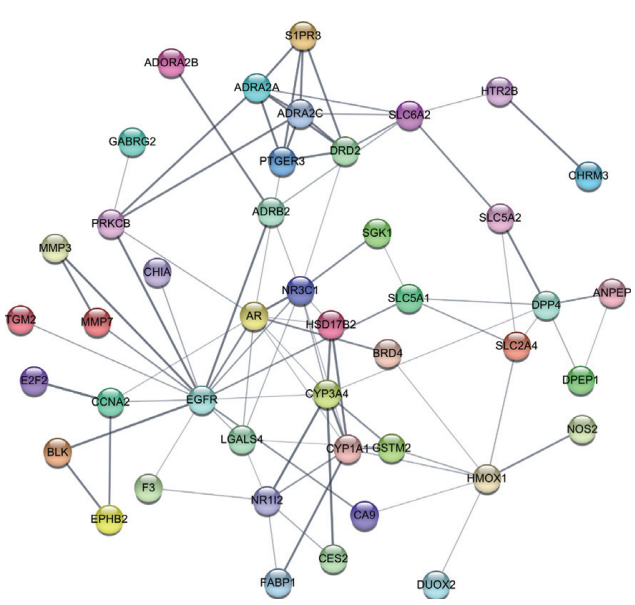

(b)

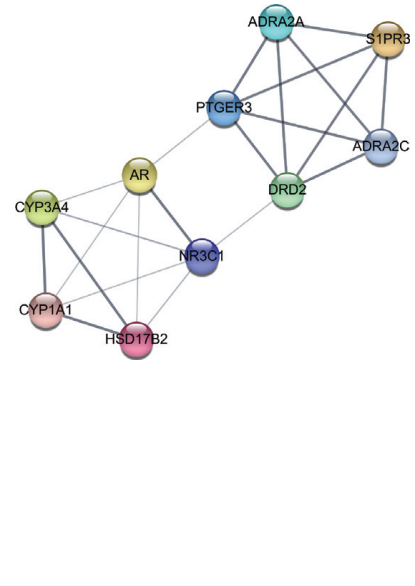

(c)

FIgUre 2: Continued. 


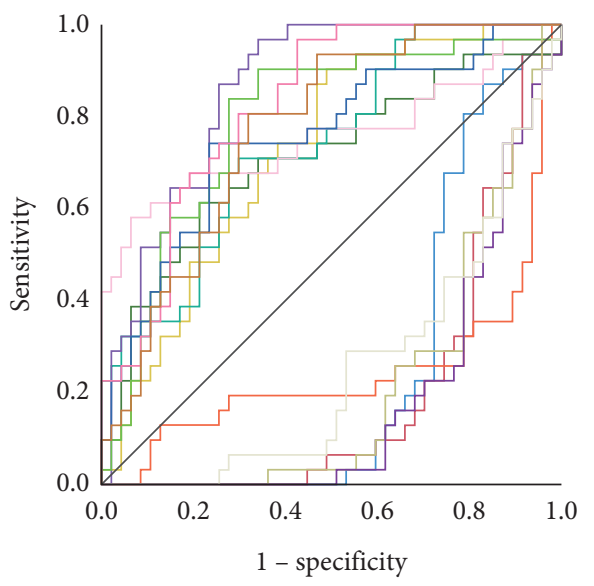

$\begin{array}{ll}\text { Protein name } & \\ - \text { EGFR } & - \text { ADRA2A } \\ - \text { AR } & - \text { ADRA2C } \\ \text { CYP3A4 } & - \text { NR1I2 } \\ - \text { NR3C1 } & - \text { SLC6A2 } \\ \text { CYP1A1 } & \text { LGALS4 } \\ \text { HMOX1 } & - \text { PTGER3 } \\ \text { DRD2 } & \text { PRKCB } \\ \text { DPP4 } & - \text { Reference line }\end{array}$

(d)

FIgURE 2: (a) Venn diagram between the targets of ARCR and the DEGs of GIN. GIN: gastric intraepithelial neoplasia; ARCR: Astragali Radix-Curcumae Rhizoma. (b) Protein-protein interaction network of the intersecting targets. Each bubble node represents a protein. The lines among inner nodes display the relationship between different proteins, and the width of lines was based on the strength of data support. (c) A core function module from the PPI network. (d) The results of ROC curve.

TABLE 2: Topology attributes of 15 potential key targets and AUC of the ROC analysis.

\begin{tabular}{|c|c|c|c|c|c|}
\hline Gene name & Protein name & Degree value & Betweenness centrality & Closeness centrality & AUC \\
\hline EGFR & Epidermal growth factor receptor & 15 & 0.4020073 & 0.5308642 & 0.254 \\
\hline $\mathrm{AR}$ & Androgen receptor & 10 & 0.16157789 & 0.48314607 & 0.202 \\
\hline CYP3A4 & Cytochrome P450 3A4 & 9 & 0.14518632 & 0.4673913 & 0.710 \\
\hline NR3C1 & Glucocorticoid receptor & 9 & 0.12596012 & 0.48314607 & 0.245 \\
\hline CYP1A1 & Cytochrome P450 1A1 & 8 & 0.08766745 & 0.43 & 0.723 \\
\hline HMOX1 & Heme oxygenase 1 & 7 & 0.11612398 & 0.37719298 & 0.733 \\
\hline DRD2 & Dopamine receptor D2 & 6 & 0.04369106 & 0.38392857 & 0.755 \\
\hline DPP4 & Dipeptidyl peptidase 4 & 6 & 0.11595851 & 0.38392857 & 0.859 \\
\hline ADRA2A & Alpha-2A adrenergic receptor & 6 & 0.01645573 & 0.3495935 & 0.787 \\
\hline ADRA2C & Alpha-2C adrenergic receptor & 6 & 0.01645573 & 0.3495935 & 0.754 \\
\hline NR1I2 & Nuclear receptor subfamily 1 group I member 2 & 6 & 0.02101245 & 0.3495935 & 0.823 \\
\hline SLC6A2 & Sodium-dependent noradrenaline transporter & 6 & 0.13161388 & 0.36752137 & 0.215 \\
\hline LGALS4 & Galectin-4 & 5 & 0.02235789 & 0.41346154 & 0.771 \\
\hline PTGER3 & Prostaglandin E2 receptor EP3 subtype & 5 & 0.03093548 & 0.37068966 & 0.185 \\
\hline PRKCB & Protein kinase $\mathrm{C}$ beta type & 5 & 0.09021468 & 0.41346154 & 0.256 \\
\hline
\end{tabular}

AUC: the area under the ROC curve.

target-KEGG pathway, involving 92 nodes and 213 edges (Figure 4). Analysis of the topology of the network showed that ARX16 (quercetin) has the highest degree value $($ degree $=18$, betweenness centrality $=0.1795$, and closeness centrality $=0.4396$ ) and indicated that quercetin is the most pivotal ingredient among the active components of ARCR in treating GIN, followed by CR4 (bisdemethoxycurcumin, degree $=13$, betweenness centrality $=0.1249$, closeness centrality $=0.3625)$, and ARX12 (kaempferol, degree $=12$, betweenness centrality $=0.0704$, closeness centrality $=0.4081)$. In the network, nine active components (CR1, CR5, ARX1, ARX3, 


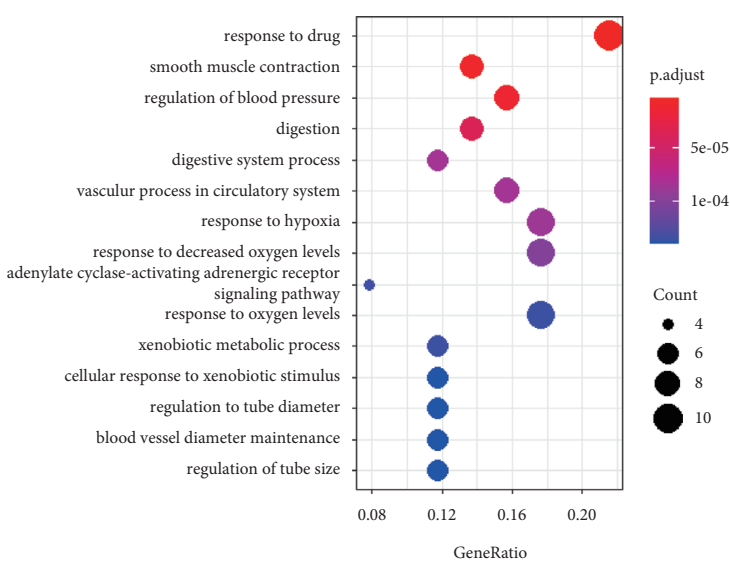

(a)

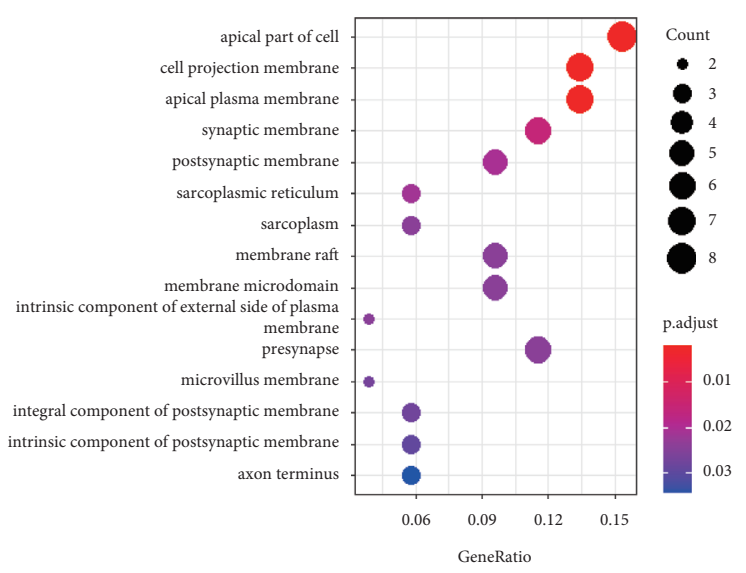

(c)

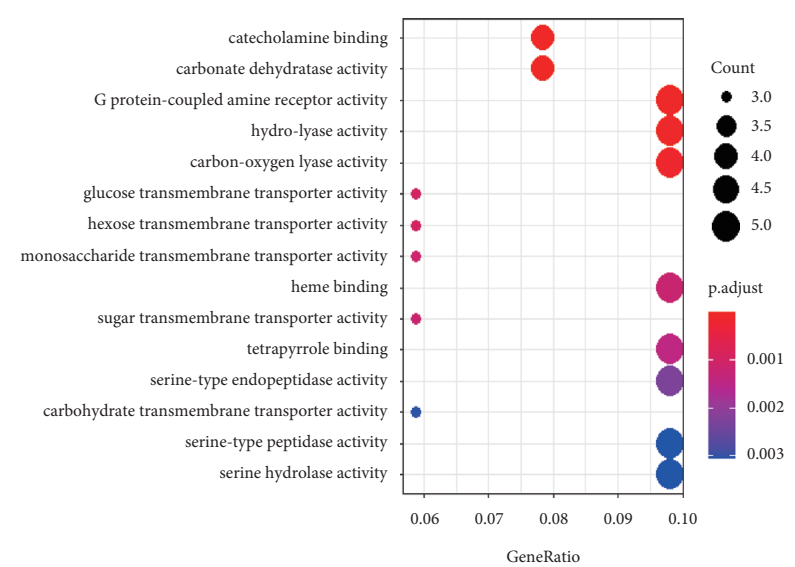

(b)

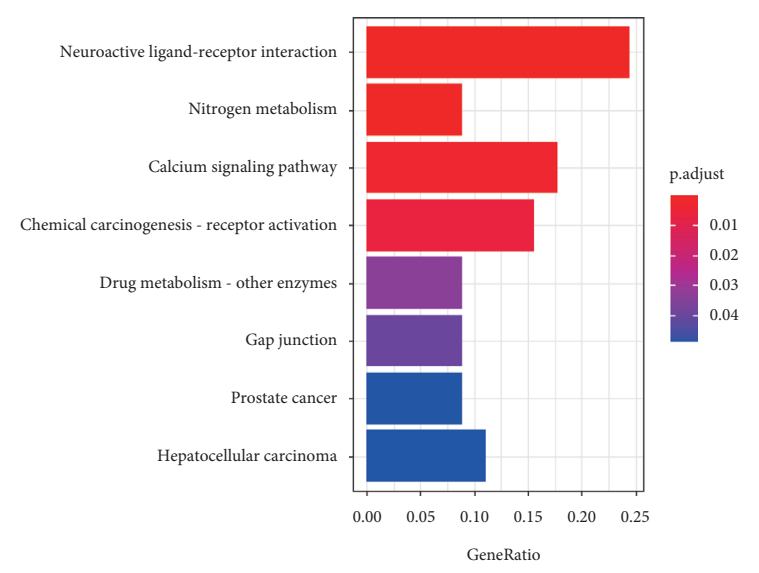

(d)

FIGURe 3: Results of GO enrichment analysis and KEGG pathway analysis of the intersecting targets. (a) Biological process; (b) molecular function; (c) cellular component; (d) Kyoto Encyclopedia of Genes and Genomes pathway analysis.

ARX7, ARX9, ARX13, ARX14, and ARX15) without intersecting targets information were deleted. Thus, a total of 22 active components of ARCR played roles in treating GIN, of which 17 were from ARX and 6 from CR (one active component was shared by ARX and CR).

3.6. Molecular Docking of Core Active Components and Key Targets. Three core active components (quercetin, bisdemethoxycurcumin, and kaempferol) and each of the corresponding key targets (CYP3A4, CYP1A1, HMOX1, DRD2, DPP4, ADRA2A, ADRA2C, NR1I2, and LGALS4) were used as ligands and receptors, respectively. When the binding energy is less than $5 \mathrm{kcal} / \mathrm{mol}$, the ligand is regarded to bind well to the receptor [30]. The molecular docking diagrams and binding energy results are shown in Figure 5.

As shown in Figure 5, all active components can bind well to their corresponding key targets, indicating quercetin, bisdemethoxycurcumin, and kaempferol are the core active components of ARCR in treating GIN, and the therapeutic effect might be mediated by nine key targets (CYP3A4, CYP1A1, HMOX1, DRD2, DPP4, ADRA2A, ADRA2C, NR1I2, and LGALS4).

\section{Discussion}

GIN is the most serious gastric precancerous lesion, and the incidence rate of GC in GIN patients is significantly higher than that in CAG and IM patients [31]. Therefore, the treatment of GIN is an effective measure to prevent GC. Previous basic studies mainly focused on different types of solid tumors (gastric cancer). The continuous process from inflammation to GIN is often ignored; however, GIN is closely related to the occurrence and development of GC. Thus, we should pay more attention to the transformation process of inflammation to GIN.

In the study, a total of 22 active components of ARCR were screened, among which three core active components (quercetin, bisdemethoxycurcumin, and kaempferol) were identified. Key pathways were mainly involved in neuroactive ligandreceptor interaction, nitrogen metabolism, calcium signaling pathway, drug metabolism, gap junction, and cancer. Moreover, in PPI network and ROC analysis, nine targets (CYP3A4, CYP1A1, HMOX1, DRD2, DPP4, ADRA2A, ADRA2C, NR1I2, and LGALS4) were selected as the key targets that may play important roles in ARCR against GIN.

Quercetin is one of the main active components of Astragali Radix, belonging to a kind of flavonoid and widely 


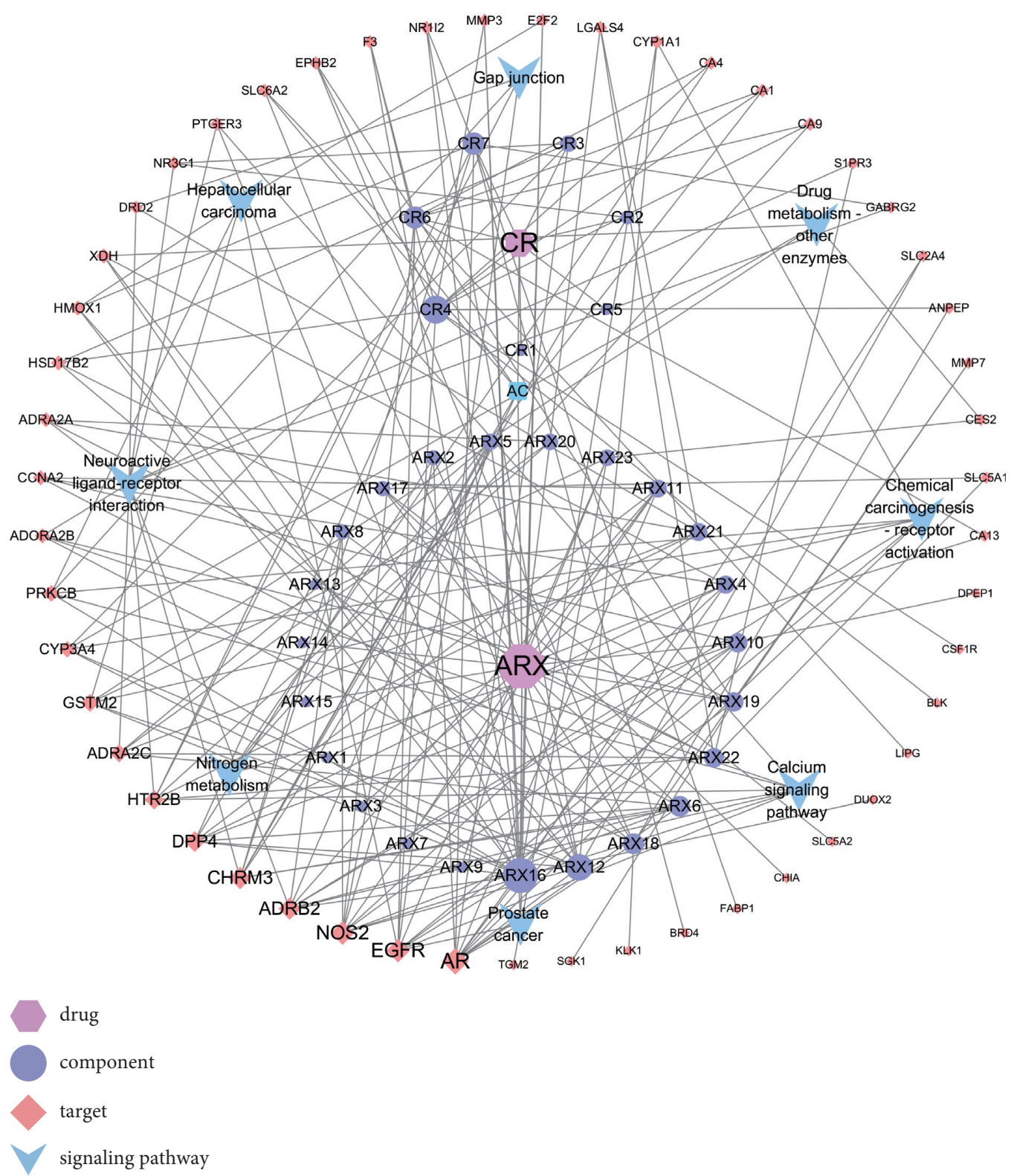

FIGURE 4: Network of ARCR-active component-intersecting target-signaling pathway. The size of the graph represents the degree value. The larger the graph, the higher the degree value. ARX16: quercetin; ARX12: kaempferol; CR4: bisdemethoxycurcumin.

found in various Chinese herbal medicines. It has anti-inflammatory effects by inhibiting the secretion of inflammatory cytokines and chemokines $[32,33]$. A populationbased study in Sweden showed that high dietary quercetin intake is inversely related to the risk of noncardia gastric adenocarcinoma and the protection appears to be particularly strong for women exposed to oxidative stress, such as tobacco smoking [34], indicating quercetin may have an important preventive effect on GC. Previous studies had further confirmed the protective effect of quercetin on gastric mucosal epithelial cells in in vivo and in vitro experiments [35-37]. As a demethoxy derivative of curcumin, bisdemethoxycurcumin is derived from Curcumae Rhizoma and has a wide range of biological characteristics. Luo et al. [38] established a human gastric adenocarcinoma xenograft model in vivo using nude mice. After intervention with bisdemethoxycurcumin, specific indicators of mitochondrial dysfunction were detected in the mitochondria: the reduction in adenosine triphosphate generation, the inner mitochondrial membrane potential, and augmentation of reactive oxygen species production and cytochrome $c$, indicating that bisdemethoxycurcumin can reduce the growth of gastric adenocarcinoma by inducing mitochondrial dysfunction. Kaempferol is a natural flavonoid that is widely existing in many fruits, vegetables, and Chinese herbal medicines [39]. Several studies have shown that a certain concentration of kaempferol can inhibit the cell cycle of a variety of tumor cells, induce tumor cell apoptosis, and thereby inhibit tumor cell/tissue invasion and metastasis [40]. A subsequent study confirmed kaempferol can activate the IRE1-JNK-CHOP signaling from cytosol to nucleus, and G9a inhibition can promote autophagic cell death in GC 


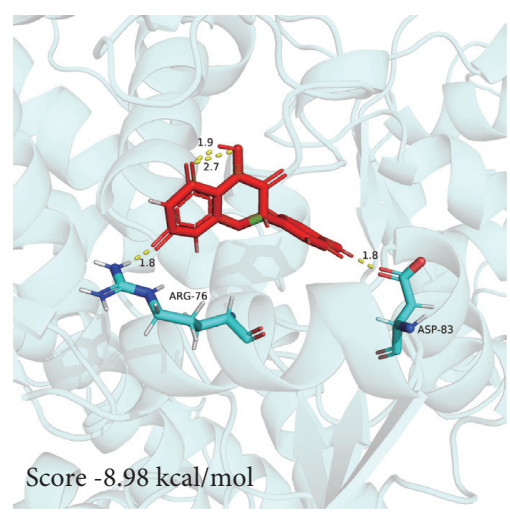

(a)

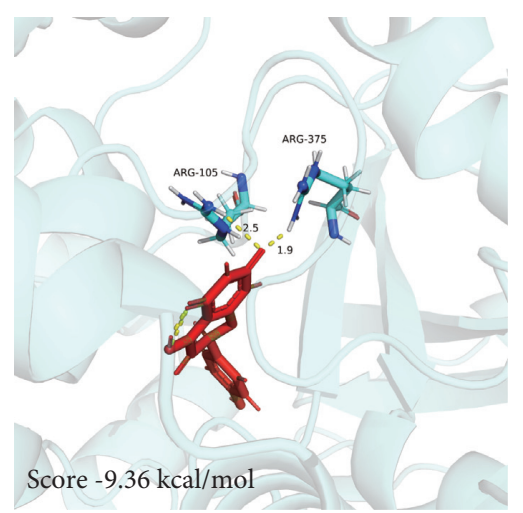

(d)

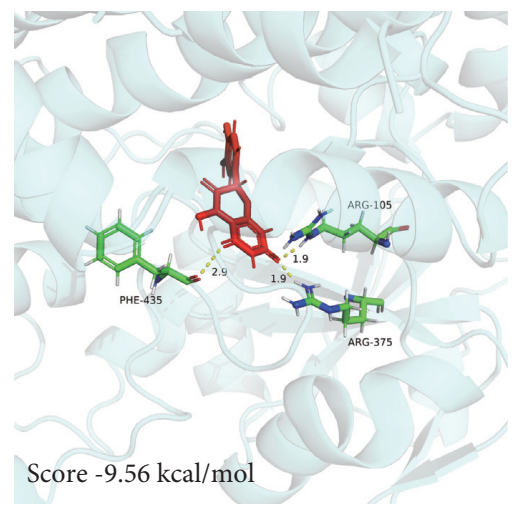

(g)

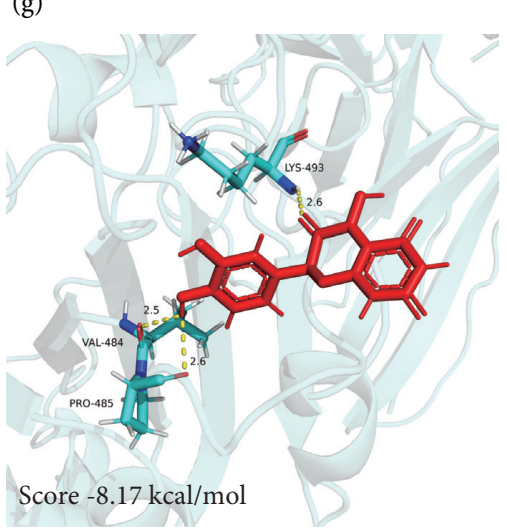

(j)

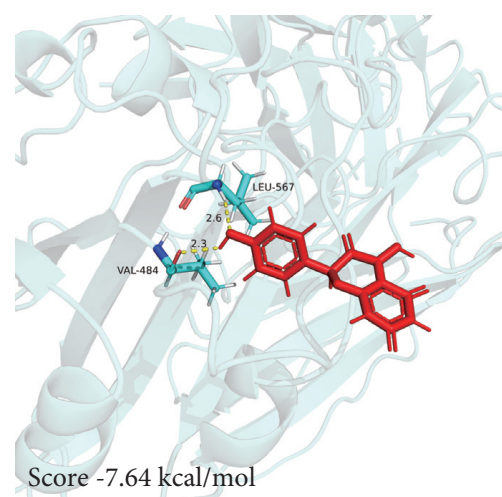

(b)

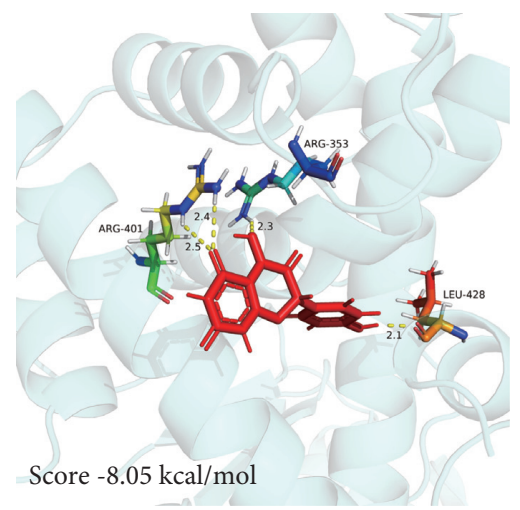

(e)

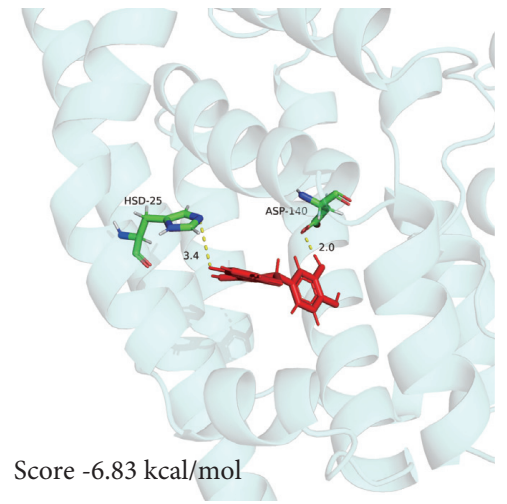

(h)

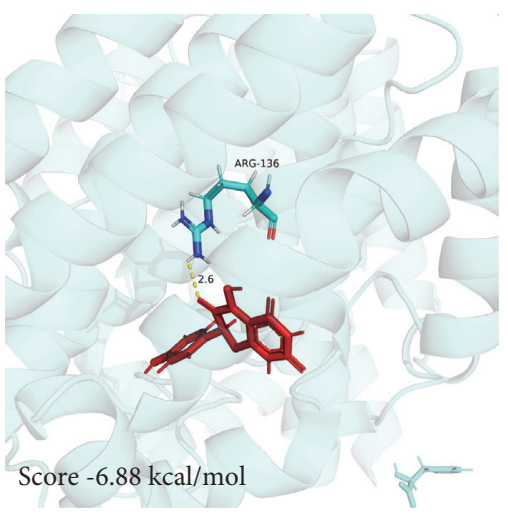

(c)

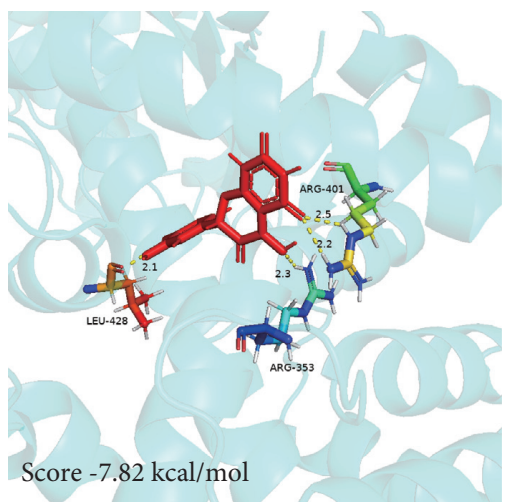

(f)

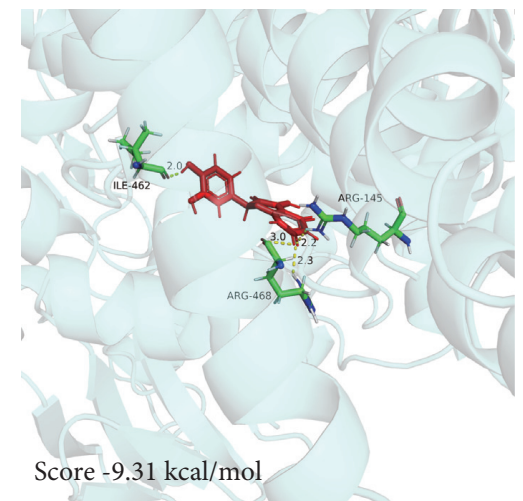

(i)

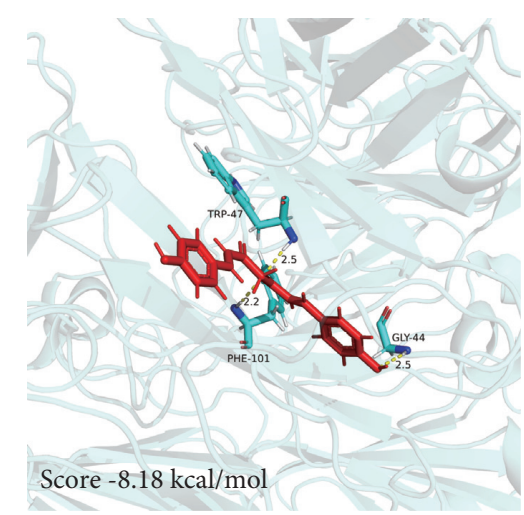

(k)

Figure 5: Molecular docking diagrams for three core active components with their corresponding key targets. (a) Kaempferol-CYP1A1; (b) kaempferol-DPP4; (c) kaempferol-HMOX1; (d) kaempferol-CYP3A4; (e) kaempferol-NR1I2; (f) quercetin-NR1I2; (g) quercetin-CYP3A4; (h) quercetin-HMOX1; (i) quercetin-CYP1A1; (j) quercetin-DPP4; and (k) bisdemethoxycurcumin-DPP4. 
cells, producing anticancer effects [41]. Previous studies confirmed that the three core active components have antigastric cancer properties; however, there is still a lack of relevant research on gastric precancerous lesions, which should be further improved.

After ROC diagnostic analysis, CYP3A4 and CYP1A1 were considered as the two key targets with the highest degree values, speculating that they may play a crucial role in ARCR against GIN. CYP3A4 and CYP1A1 are members of the cytochrome P450 superfamily of enzymes. A recent study found that the expression of CYP3A4 might be related to the potential carcinogenic transformation of CAG to GC, which may be biomarkers to predict the progression of $\mathrm{CAG}$ and the poor prognosis of GC [42]. As an isoenzyme of the CYP family I, CYP1A1 mainly participates in the oxidative conversion of the xenobiotics. The importance of CYP1A1 derives from its preferential extrahepatic activities and widespread substrate specificity mainly of xenobiotics [43]. However, its metabolic reactions may accidentally lead to producing the highly reactive compounds that form DNA adducts, contributing to mutagenesis and carcinogenesis $[44,45]$. The neuroactive ligand-receptor interaction pathway is the most significantly enriched signaling pathway, involving three key targets (DRD2, ADRA2A, and ADRA2C). G protein-coupled receptors (GPCRs) are large and diverse signaling receptors that are altered in various cancers and regulate cancer progression. Dopamine receptors, a class of GPCRs, have 5 subtypes named DRD1-DRD5 [46]. In general, dopamine receptors are involved in neurological disorders; however, studies indicated that dopamine receptors are also involved in tumor progression [47-49]. Previous studies suggested that dopamine receptor D2 (DRD2) expression can be used to evaluate the prognosis of patients with gastric or esophageal cancer $[49,50]$. Moreover, targeting DRD2 has been a novel therapeutic strategy in tumor-related diseases [51-53]. Thus, DRD2 may play an important role in GC. However, no previous studies provided experimental evidence of how DRD2 functions in GIN. ADRA2A and ADRA2C are the alpha-2-adrenergic receptors, a type of adrenergic receptors, and also are the paralogs of DRD2. Studies have confirmed that the expression of ADRA2A is related to the risk of breast cancer $[54,55]$. Therefore, these key targets may become candidate genes for the treatment of GIN.

The study still has several limitations. First, the results of the study are not verified in experiments. However, the study is the first to explore the mechanism of ARCR in the treatment of GIN via network pharmacology and molecular docking and will provide theoretical references for subsequent experiments in proven models. Second, the quality of the microarray expression profiling dataset could not be evaluated. However, the ROC diagnostic analysis was used to verify the key targets, which improved the stability and feasibility of the results.

\section{Conclusions}

In summary, the study used network pharmacology and molecular docking to systematically elaborate the mechanism of ARCR in the treatment of GIN. The results indicated that quercetin, bisdemethoxycurcumin, and kaempferol are the core active components, which may generate therapeutic effects on GIN through targets named CYP3A4, CYP1A1, HMOX1, DRD2, DPP4, ADRA2A, ADRA2C, NR1I2, and LGALS4. The study revealed the material basis of ARCR and the possible mechanism for the treatment of GIN and unearthed the potential targets of ARCR in the treatment of GIN, which will provide a reference for follow-up experimental research.

\section{Abbreviations}

ARCR: Astragali Radix-Curcumae Rhizoma

GIN: Gastric intraepithelial neoplasia

PPI: $\quad$ Protein-protein interaction

ROC: Receiver operating characteristic curve

GO: Gene Ontology

BP: Biological process

MF: Molecular function

CC: Cellular component

KEGG: Kyoto Encyclopedia of Genes and Genomes

IGC: Intestinal gastric cancer

CAG: Chronic atrophic gastritis

ARX: Astragali Radix

CR: Curcumae Rhizoma

TCMSP: Traditional Chinese Medicine Database and Analysis Platform

OB: Oral bioavailability

DL: Drug-likeness

GEO: Gene Expression Omnibus

AUC: The area under the ROC curve.

\section{Data Availability}

The data used in the study are available from the corresponding author upon request.

\section{Conflicts of Interest}

The authors declare that there are no conflicts of interest regarding the publication of this article.

\section{Acknowledgments}

This work was supported by the National Natural Science Foundation of China (no. 81703943) and Jiangsu Province Postgraduate Cultivation Innovation Project-Graduate Research and Practice Innovation Program (no. SJCX21_0773).

\section{Supplementary Materials}

Supplementary Table 1: Differentially expressed genes of GIN in GSE130823. (Supplementary Materials)

\section{References}

[1] H. Sung, J. Ferlay, R. L. Siegel et al., "Global cancer statistics 2020: GLOBOCAN estimates of incidence and mortality 
worldwide for 36 cancers in 185 countries," CA: A Cancer Journal for Clinicians, vol. 71, no. 3, pp. 209-249, 2021.

[2] J. Han, Z. Nie, P. Li et al., "Comparison of treatment modalities for locally advanced gastric cancer: a propensity score matching analysis," Journal of Cancer, vol. 11, no. 15, pp. 4421-4430, 2020.

[3] W. Chen, R. Zheng, P. D. Baade et al., "Cancer statistics in China, 2015," CA: A Cancer Journal for Clinicians, vol. 66, no. 2, pp. 115-132, 2016.

[4] P. Laurén, "The two histological main types of gastric carcinoma: diffuse and so-called intestinal-type carcinoma," Acta Pathologica et Microbiologica Scandinavica, vol. 64, no. 1, pp. 31-49, 1965.

[5] P. Correa, "Human gastric carcinogenesis: a multistep and multifactorial process--first American cancer society award lecture on cancer epidemiology and prevention," Cancer Research, vol. 52, no. 24, pp. 6735-6740, 1992.

[6] R. L. Sun, D. C. Tang, and J. F. Gu, "Study on intervention effect of Astragali Radix-Curcumae Rhizoma on growth and metastasis of colon cancer in orthotopic transplantation mice model of colon cancer," Zhongguo Zhongyao Zazhi, vol. 46, no. 9, pp. 2267-2275, 2021.

[7] W. H. Zang, D. C. Tang, G. Yin, and B. B. Li, "Inhibition effects of compatibility of Astragali Radix and curcumae rhizoma and combination with cisplatin on orthotopic tumor in nude mice bearing human hepatocellular carcinoma," Zhongguo Shi Yan Fang Ji Xue Za Zhi, vol. 20, no. 5, pp. 131-136, 2014.

[8] S. T. Qian, J. S. Yu, and Z. Y. Chen, "Effects of Yiqi Huoxue Decoction on the expression of Sonic Hedgehog signaling pathway in the stomach mucosa of rats with gastric precancerous lesions," Zhonghua Zhong Yi Yao Za Zhi, vol. 36, no. 1, pp. 446-451, 2021.

[9] Y. Z. Wang, Y. R. Ma, Y. S. Liu, Y. L. Si, J. B. Zhang, and L. Sun, "Clinical effects of Yiqi Huoxue Fang combined with quadruple therapy on Helicobacter pylori positive chronic atrophic gastritis and its effects on pepsinogen," World Chinese Medicine, vol. 13, no. 2, pp. 325-328, 2018.

[10] L. Zhang, H. Shen, and X. B. Zhou, "Treatment of chronic atrophic gastritis precancerous lesions from theory of qi deficiency and blood stasis," Modern Chinese Clinical Medicine, vol. 20, no. 1, pp. 16-20, 2013.

[11] J. Ru, P. Li, J. Wang et al., "TCMSP: a database of systems pharmacology for drug discovery from herbal medicines," Journal of Cheminformatics, vol. 6, no. 13, p. 13, 2014.

[12] D. Gfeller, A. Grosdidier, M. Wirth, A. Daina, O. Michielin, and V. Zoete, "SwissTargetPrediction: a web server for target prediction of bioactive small molecules," Nucleic Acids Research, vol. 42, pp. W32-W38, 2014.

[13] Y. Wang, J. Xiao, T. O. Suzek, J. Zhang, J. Wang, and S. H. Bryant, "PubChem: a public information system for analyzing bioactivities of small molecules," Nucleic Acids Research, vol. 37, pp. W623-W633, 2009.

[14] UniProt, "The universal protein knowledgebase in 2021," Nucleic Acids Research, vol. 49, pp. D480-d489, 2021.

[15] T. Barrett, S. E. Wilhite, P. Ledoux et al., "NCBI GEO: archive for functional genomics data sets-update," Nucleic Acids Research, vol. 41, pp. D991-D995, 2013.

[16] M. E. Ritchie, B. Phipson, D. Wu et al., "Limma powers differential expression analyses for RNA-sequencing and microarray studies," Nucleic Acids Research, vol. 43, no. 7, p. e47, 2015.

[17] C. Y. Li, J.-H. Cai, J. J. P. Tsai, and C. C. N. Wang, "Identification of hub genes associated with development of head and neck squamous cell carcinoma by integrated bioinformatics analysis," Frontiers in Oncology, vol. 10, p. 681, 2020.

[18] D. Szklarczyk, A. L. Gable, D. Lyon et al., "STRING v11: protein-protein association networks with increased coverage, supporting functional discovery in genome-wide experimental datasets," Nucleic Acids Research, vol. 47, pp. D607d613, 2019.

[19] P. Shannon, A. Markiel, and O. Ozier, "Cytoscape: a software environment for integrated models of biomolecular interaction networks," Genome Research, vol. 13, no. 11, pp. 2498-2504, 2003.

[20] G. D. Bader and C. W. Hogue, "An automated method for finding molecular complexes in large protein interaction networks," BMC Bioinformatics, vol. 4, p. 2, 2003.

[21] C. H. Chin, S. H. Chen, H. H. Wu, C. W. Ho, M. T. Ko, and C. Y. Lin, "cytoHubba: identifying hub objects and subnetworks from complex interactome," BMC Systems Biology, vol. 8, p. S11, 2014.

[22] G. Yu, L.-G. Wang, Y. Han, and Q.-Y. He, "clusterProfiler: an $\mathrm{R}$ package for comparing biological themes among gene clusters," OMICS: A Journal of Integrative Biology, vol. 16, no. 5, pp. 284-287, 2012.

[23] T. Sterling and J. J. Irwin, "Zinc 15-ligand discovery for everyone," Journal of Chemical Information and Modeling, vol. 55, no. 11, pp. 2324-2337, 2015.

[24] J. L. Sussman, D. Lin, J. Jiang et al., "Protein Data Bank (PDB): database of three-dimensional structural information of biological macromolecules," Acta Crystallographica. Section D, Biological Crystallography, vol. 54, pp. 1078-1084, 1998.

[25] A. Grosdidier, V. Zoete, and O. Michielin, "SwissDock, a protein-small molecule docking web service based on EADock DSS," Nucleic Acids Research, vol. 39, pp. W270W277, 2011.

[26] H. M. Ni, Z. Y. Dong, and X. M. Chen, "Research progress of the curative effects of main active components of Astragalus membranaceus in treatment of diabetic nephropathy," Jie Fang Jun Yi Xue Za Zhi, vol. 46, no. 3, pp. 294-299, 2021.

[27] T.-J. Zhao, P.-Y. Fu, L.-L. Liu, Y. Zheng, and Y. Peng, "Effects of several active ingredients of Rhizoma curcumae on liver diseases," World Chinese Journal of Digestology, vol. 25, no. 27, pp. 2433-2440, 2017.

[28] J. F. Wu, Y. Z. Yao, G. Yin, W. W. Tao, D. C. Tang, and W. H. Zang, "HPLC analysis of single, merging, and boiled together in Astragali Radix and Curcumae Rhizoma," Zhonghua Zhong Yi Yao Za Zhi, vol. 33, no. 4, pp. 1567-1570, 2018.

[29] J. Xie, Y. X. Y. Gong, L. S. Ding, P. Luo, and L. S. Qing, "Progress in clinical and pharmacological studies of Astragali Radix and its active components against sepsis," Zhong Cao Yao, vol. 52, no. 8, pp. 2502-2510, 2021.

[30] L. Guo, H. Shi, and L. Zhu, "Siteng Fang reverses multidrug resistance in gastric cancer: a network pharmacology and molecular docking study," Frontiers in Oncology, vol. 11, Article ID 671382, 2021.

[31] A. C. de Vries, N. C. T. van Grieken, C. W. N. Looman et al., "Gastric cancer risk in patients with premalignant gastric lesions: a nationwide cohort study in The Netherlands," Gastroenterology, vol. 134, no. 4, pp. 945-952, 2008.

[32] Y. Li, J. Yao, C. Han et al., "Quercetin, inflammation and immunity," Nutrients, vol. 8, no. 3, p. 167, 2016.

[33] Y. Edo, A. Otaki, and K. Asano, "Quercetin enhances the thioredoxin production of nasal epithelial cells in vitro and in vivo," Medicine, vol. 5, no. 4, p. 124, 2018. 
[34] A. M. Ekström, M. Serafini, O. Nyrén, A. Wolk, C. Bosetti, and R. Bellocco, "Dietary quercetin intake and risk of gastric cancer: results from a population-based study in Sweden," Annals of Oncology, vol. 22, no. 2, pp. 438-443, 2011.

[35] K. Wang, R. Liu, J. Li et al., "Quercetin induces protective autophagy in gastric cancer cells: involvement of Akt-mTORand hypoxia-induced factor $1 \alpha$-mediated signaling," Autophagy, vol. 7, no. 9, pp. 966-978, 2011.

[36] S. Chakraborty, S. Stalin, N. Das, S. Thakur Choudhury, S. Ghosh, and S. Swarnakar, "The use of nano-quercetin to arrest mitochondrial damage and MMP-9 upregulation during prevention of gastric inflammation induced by ethanol in rat," Biomaterials, vol. 33, no. 10, pp. 2991-3001, 2012.

[37] R. González-Segovia, J. L. Quintanar, E. Salinas, R. CeballosSalazar, F. Aviles-Jiménez, and J. Torres-López, "Effect of the flavonoid quercetin on inflammation and lipid peroxidation induced by Helicobacter pylori in gastric mucosa of Guinea pig," Journal of Gastroenterology, vol. 43, no. 6, pp. 441-447, 2008.

[38] C. Luo, Z. Du, X. Wei, G. Chen, and Z. Fu, "Bisdemethoxycurcumin attenuates gastric adenocarcinoma growth by inducing mitochondrial dysfunction," Oncology Letters, vol. 9, no. 1, pp. 270-274, 2015.

[39] M. Bajpai, A. Pande, S. K. Tewari, and D. Prakash, "Phenolic contents and antioxidant activity of some food and medicinal plants," International Journal of Food Sciences \& Nutrition, vol. 56, no. 4, pp. 287-291, 2005.

[40] E. Jo, S. J. Park, Y. S. Choi, W.-K. Jeon, and B.-C. Kim, "Kaempferol suppresses transforming growth factor- $\beta 1$-induced epithelial-to-mesenchymal transition and migration of A549 lung cancer cells by inhibiting akt1-mediated phosphorylation of Smad3 at threonine-179," Neoplasia, vol. 17, no. 7, pp. 525-537, 2015.

[41] T. W. Kim, S. Y. Lee, M. Kim, C. Cheon, and S.-G. Ko, "Kaempferol induces autophagic cell death via IRE1-JNKCHOP pathway and inhibition of G9a in gastric cancer cells," Cell Death \& Disease, vol. 9, no. 9, p. 875, 2018.

[42] F. Zhang, F. Wang, C. Chen et al., "Prediction of progression of chronic atrophic gastritis with Helicobacter pylori and poor prognosis of gastric cancer by CYP3A4," Journal of Gastroenterology and Hepatology, vol. 35, no. 3, pp. 425-432, 2020.

[43] L. Sadeghi-Amiri, A. Barzegar, N. Nikbakhsh-Zati, and P. Mehraban, "Hypomethylation of the XRE -1383 site is associated with the upregulation of CYP1A1 in gastric adenocarcinoma," Gene, vol. 769, 2021.

[44] M. Kubota, K. Sogawa, Y. Kaizu et al., "Xenobiotic responsive element in the $5^{\prime}$-upstream region of the human P-450c Gene1," Journal of Biochemistry, vol. 110, no. 2, pp. 232-236, 1991.

[45] J. P. Whitlock Jr., "Induction of cytochrome P4501A1," Annual Review of Pharmacology and Toxicology, vol. 39, no. 1, pp. 103-125, 1999.

[46] H. Lee, S. Shim, and J. S. Kong, "Overexpression of dopamine receptor D2 promotes colorectal cancer progression by activating the $\beta$-catenin/ZEB1 axis," Cancer Science, vol. 112, pp. 1-12, 2021.

[47] M. Pornour, G. Ahangari, S. Hejazi, and A. Deezagi, "New perspective therapy of breast cancer based on selective dopamine receptor D2 agonist and antagonist effects on MCF-7 cell line," Recent Patents on Anti-cancer Drug Discovery, vol. 10, no. 2, pp. 214-223, 2015.

[48] V. V. Prabhu, N. S. Madhukar, C. Gilvary et al., "Dopamine receptor D5 is a modulator of tumor response to dopamine receptor D2 antagonism," Clinical Cancer Research, vol. 25, no. 7, pp. 2305-2313, 2019.

[49] J. Mu, W. Huang, Z. Tan et al., "Dopamine receptor D2 is correlated with gastric cancer prognosis," Oncology Letters, vol. 13, no. 3, pp. 1223-1227, 2017.

[50] L. Li, M. Miyamoto, and Y. Ebihara, "DRD2/DARPP-32 expression correlates with lymph node metastasis and tumor progression in patients with esophageal squamous cell carcinoma," World Journal of Surgery, vol. 30, no. 9, pp. 16721679, 2006.

[51] S. R. Pierce, Z. Fang, Y. Yin et al., "Targeting dopamine receptor D2 as a novel therapeutic strategy in endometrial cancer," Journal of Experimental \& Clinical Cancer Research, vol. 40, no. 1, p. 61, 2021.

[52] R. Bakadlag, P. Jandaghi, J. D. Hoheisel, and Y. Riazalhosseini, "The potential of dopamine receptor D2 (DRD2) as a therapeutic target for tackling pancreatic cancer," Expert Opinion on Therapeutic Targets, vol. 23, no. 5, pp. 365-367, 2019.

[53] W. Hu, L. Zhang, S. Ferri-Borgogno et al., "Targeting dopamine receptor D2 by imipridone suppresses uterine serous cancer malignant phenotype," Cancers, vol. 12, no. 9, p. 2436, 2020.

[54] M. Y. Shkurnikov, V. V. Galatenko, A. E. Lebedev, V. E. Podol'skii, E. A. Tonevitskii, and D. V. Mal'tseva, "On statistical relationship between ADRA2A expression and the risk of breast cancer relapse," Bulletin of Experimental Biology and Medicine, vol. 157, no. 4, pp. 454-458, 2014.

[55] B. Kaabi, G. Belaaloui, W. Benbrahim et al., "ADRA2A germline gene polymorphism is associated to the severity, but not to the risk, of breast cancer," Pathology and Oncology Research, vol. 22, no. 2, pp. 357-365, 2016. 